\title{
Dose-volume histogram prediction using density estimation
}

Johanna Skarpman Munter and Jens Sjölund

\author{
Linköping University Post Print
}

\section{Tweet}

N.B.: When citing this work, cite the original article.

Original Publication:

Johanna Skarpman Munter and Jens Sjölund, Dose-volume histogram prediction using density estimation, 2015, Physics in Medicine and Biology, (60), 17, 6923-6936.

http://dx.doi.org/10.1088/0031-9155/60/17/6923

Copyright: IOP Publishing: Hybrid Open Access

http://www.iop.org/

Postprint available at: Linköping University Electronic Press

http://urn.kb.se/resolve?urn=urn:nbn:se:liu:diva-121747 


\title{
Dose-Volume Histogram Prediction using Density Estimation
}

\author{
Johanna Skarpman Munter ${ }^{1}$ and Jens Sjölund*1, 2, 3 \\ ${ }^{1}$ Elekta Instrument AB, Kungstensgatan 18, Box 7593, SE-103 93 Stockholm, Sweden \\ ${ }^{2}$ Center for Medical Image Science and Visualization (CMIV), Linköping University, Sweden \\ ${ }^{3}$ Department of Biomedical Engineering, Linköping University, Linköping, Sweden
}

June 25, 2015

\begin{abstract}
Knowledge of what dose-volume histograms that can be expected for a previously unseen patient could increase consistency and quality in radiotherapy treatment planning. We propose a machine learning method that uses previous treatment plans to predict such a dose-volume histogram. Key to the approach is the framing of dose-volume histograms in a probabilistic setting.

The training consists of estimating, from the patients in the training set, the joint probability distribution of some predictive features and the dose. The joint distribution immediately provides an estimate of the conditional probability of the dose given values of the predictive features. The prediction consists of estimating, from the new patient, the distribution of the predictive features and marginalizing the conditional probability from the training over this. Integrating the resulting probability distribution for the dose yields the estimate of the dose-volume histogram.

To illustrate how the proposed method relates to previously proposed methods, we use the signed distance to the target boundary as a single predictive feature. As a proof-of-concept, we predicted dose-volume histograms for the brainstem of 22 acoustic schwannoma patients treated with stereotactic radiosurgery, and for the lung of 9 lung cancer patients treated with stereotactic body radiation therapy. Comparing with two previous attempts at dose-volume histogram prediction we find that, given the same input data, the predictions are similar.

In summary, we propose a method for dose-volume histogram prediction that exploits the intrinsic probabilistic properties of dose-volume histograms. We argue that the proposed method makes up for some deficiencies in the previously proposed methods, thereby potentially increasing ease of use, flexibility and ability to perform well with small amounts of training data.
\end{abstract}

*Corresponding author. E-mail: jens.sjolund@liu.se 


\section{Introduction}

A third of the women and half of the men in the United States are expected to develop cancer in his or her lifetime [22], a large fraction of which will receive radiotherapy. The goal of a radiotherapy treatment is, typically, to deliver a sufficiently high dose to the target while sparing healthy tissue. Treatment planning requires striking a balance between such conflicting objectives, taking into account their individual importance. This is a delicate and time-consuming task that grows even more complex as the number of organs at risk (OARs) increases.

The criteria for clinically acceptable plans are often derived from populationbased data, such as the Radiation Therapy Oncology Group (RTOG) guidelines. While appropriate for some patients, such criteria fail to account for the geometry of individual patients and can sometimes be either easily surpassed or too restrictive. Through skill and experience, planners can learn to recognize such cases - provided that they are given enough time. Tools such as multicriteria optimization can help users articulate and prioritize between conflicting objectives. Nevertheless, the final set of priorities is ultimately decided by the planner. Indeed, several studies have shown that the quality of Intensity Modulated Radiation Therapy (IMRT) plans vary both between planners and between treatment centers $[4,10,12]$. These variations can be so great that the risk of radiation-induced complications is markedly increased [19]. On a fundamental level, these variations exist because it is difficult to compare plans for different patients. As a consequence it is a futile task for any human to harness all of the knowledge from previous, carefully developed, treatment plans. We therefore argue that a method that can predict plans based on a set of previously created plans can be an important tool for increasing conformity and thereby also quality of treatment plans.

One such method [8] retrieves similar patient cases by matching the beam's eye view projections of the structure contours. This has been successfully used to transfer clinical expertise between clinics [13]. However, because it relies on $2 \mathrm{D}$ projections of the anatomy from a fixed set of angles, the method is sensitive to patient setup and limits the possibility of tailoring radiation delivery.

Another approach is to use the overlap volume histogram $(\mathrm{OVH})[14,25]$, that describes the volume of an OAR that is within a certain distance from the target boundary. The OVH can be used for quality assurance by using a point on the OVH to query a database of plans for cases where the distances between OAR and target are less than or equal to the present case at the same time as the dose is lower [25]. The dose-volume histograms (DVHs) retrieved in this way can also be used to formulate realistic DVH-objectives for the treatment plan optimization [26].

Although DVH-objectives are notoriously difficult to handle in the optimization process [11], progress towards an efficient reformulation of the problem have been made [27]. Since DVHs are widely used for clinical assessment of dose plans, it would have an immediate effect - both for treatment plan optimization and quality assurance - if it were possible to predict what DVHs that are achievable. 
Appenzoller et al. [1] proposed a two-step parametric approach to DVH prediction that, similarly to the $\mathrm{OVH}$, uses the distance from a voxel to the target boundary. The resulting model can be used to predict DVHs for a new patient and a clinical implementation has demonstrated its ability to detect suboptimal plans [18]. A central assumption is, however, that a particular set of parameterizations can be found that accurately captures the relevant features. As will be shown in this work, this is an unnecessary restriction.

On the contrary, a study by Zhu et al. [28] that used Support Vector Regression (SVR) to generate DVHs for OARs from data consisting of target size, OAR sizes and OVHs, makes too few assumptions - and does not always capture some of the characteristics native to the problem. As established [29], a DVH can be interpreted in terms of a cumulative distribution function, whereby it must be non-increasing and take values only in the range $[0,1]$. Since the problem is approached as one of pure regression in [28], unallowable predictions may result.

We propose a method for DVH prediction that exploits the probabilistic interpretation of a DVH [29], thereby ensuring that the predicted DVHs fulfill the mathematical definition of a DVH, while making efficient use of the input data with minimal assumptions.

\section{Methods and Materials}

In general terms, our method uses past treatments to learn the relationship between the dose and other features. This relationship is then used to predict the expected dose probability distribution of a new patient for which we want to predict a DVH. The DVH is calculated from the dose probability distribution based on a probabilistic interpretation of a DVH [29] that will be further described in the next section.

\subsection{Probabilistic Interpretation of a DVH}

A cumulative probability distribution is defined as $P(D \leq d)=\int_{-\infty}^{d} p_{D}(s) \mathrm{d} s$. In our case, the value $d$ is the dose, $P(D \leq d)$ is the probability that a random variable $D$ is less than or equal to the dose $d$ and $p_{D}(d)$ is the probability distribution for the dose in the considered volume.

A DVH is defined as the volume ratio that receives a radiation dose that is higher than or equal to a certain value. It can be interpreted as the probability that a randomly chosen point in the considered volume receives a certain dose or higher. This means that - in analogy with the cumulative probability distribution - we can write the DVH as a function of the dose $d$ as the probability that a random variable $D$ is larger than or equal to $d$ as $\operatorname{DVH}(d)=P(D \geq$ $d)=1-P(D \leq d)$. So if we know the probability distribution of the dose we can determine the DVH by

$$
\operatorname{DVH}(d)=1-P(D \leq d)=1-\int_{0}^{d} p_{D}(s) \mathrm{d} s, \quad d \geq 0 .
$$


The following section will describe how to make a prediction of the dose probability distribution.

\subsection{Algorithm Outline}

If we estimate the joint probability distribution $p_{X_{1}, \ldots, X_{n}}\left(x_{1}, \ldots, x_{n}\right)$ for a set of $n$ variables $x_{1}, \ldots, x_{n}$, we can find the probability distribution for a single one of them by marginalizing over the others. This can be turned into a machine learning algorithm by first learning $p_{X_{1}, \ldots, X_{n}}\left(x_{1}, \ldots, x_{n}\right)$ from training data. In the test case we can then infer the probability distribution for one of the variables based on the distribution (in the test data) of the other variables. As a special case, let $x_{1}=d$ be the dose in each voxel. This is the primary variable of interest in this work and we want to relate that to other input information $x_{2}, \ldots, x_{n}$ that we have about the voxel. By predicting the probability distribution of the dose, we can calculate a DVH according to (1). Algorithm 1 gives a detailed description of the training phase of the DVH prediction. It provides an estimate of $p_{D \mid X_{2}, \ldots, X_{n}}\left(d \mid x_{2}, \ldots x_{n}\right)$, from which predictions can be made by marginalizing over the probability distribution for the input data $p_{X_{2}, \ldots, X_{n}}^{*}\left(x_{2}, . . x_{n}\right)$ of the test patient (superscript * denotes that the probability distribution refers to the test patient and not to the training set). Algorithm 2 describes the prediction part of the algorithm.

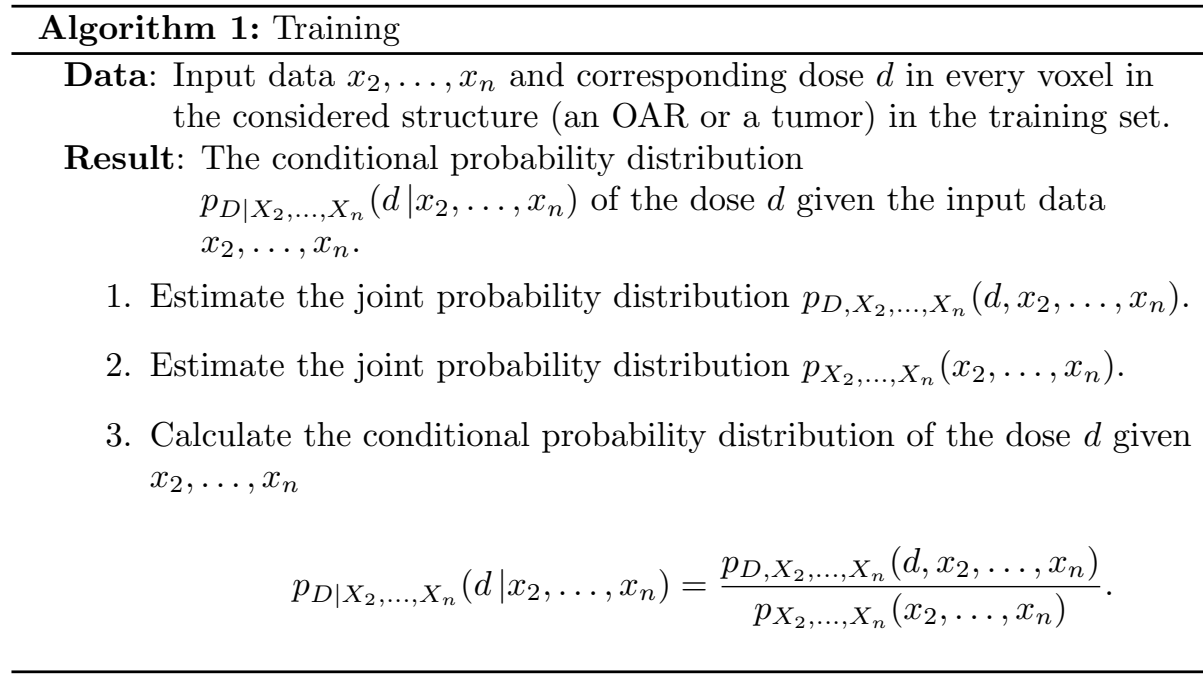




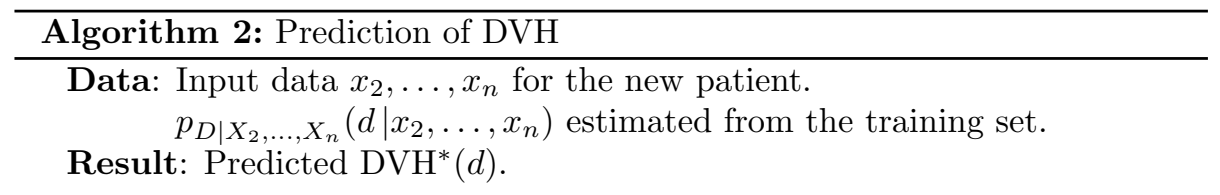

1. Estimate the probability distribution $p_{X_{2}, \ldots, X_{n}}^{*}\left(x_{2}, \ldots, x_{n}\right)$ for the new patient.

2. Marginalize over $p_{X_{2}, \ldots, X_{n}}^{*}\left(x_{2}, \ldots, x_{n}\right)$ to retain a prediction of the distribution $p_{D}^{*}(d)$

$$
p_{D}^{*}(d)=\int_{x_{2, \ldots, n}} p_{D \mid X_{2}, \ldots, X_{n}}\left(d \mid x_{2}, \ldots, x_{n}\right) p_{X_{2}, \ldots, X_{n}}^{*}\left(x_{2}, \ldots, x_{n}\right) \mathrm{d} x_{2, \ldots, n}
$$

3. Calculate $\mathrm{DVH}^{*}(d)$ using equation (1).

$$
\mathrm{DVH}^{*}(d)=1-\int_{0}^{d} p_{D}^{*}(s) \mathrm{d} s
$$

\subsection{Density estimation}

The probability distribution estimates can be obtained in a multitude of ways, e.g. using a Gaussian mixture model [20], density estimation trees [21] or kernel density estimation [3, 20]. The two latter are non-parametric methods, meaning that no underlying distribution is assumed. Parametric methods like Gaussian mixture models require more assumptions on the data to perform well [21, 23]. Due to higher accuracy [21] and less implementation effort [23] we choose to use kernel density estimation over density estimation trees. However, the proposed algorithm is agnostic to the specifics of the density estimation.

Kernel density estimation can in short be described as applying a kernel to each sample point and then summing the kernels together to generate a continuous estimate of a distribution. A common choice of kernel that has also been used in our implementation is the Gaussian kernel. However, the bandwidth parameter has to be decided, which is not trivial in general. In our implementation all probability densities have been estimated using an automatic data driven procedure for bandwidth determination [5].

\subsection{Training Data}

As mentioned before, we demonstrate our method by using kernel density estimation, where we relate voxel dose to a single feature. To allow comparison with previous work $[1,25,28]$ we take this feature to be the signed distance to the target boundary. That the distances are signed means that negative distances 
account for voxels that overlap with the target volume. The target boundarywhere the distance is zero-is defined as the voxels that are 6 -connected $[9,24]$ to the voxels outside the target volume. 6 -connectedness means that the voxels are connected to their faces, but not to the edges. The signed distance field can be calculated efficiently through a so called distance transform $[16,17]$. Incidentally, the previously mentioned OVH that has been used in previous studies $[1,25,28]$ is the cumulative distribution of the signed distances in a volume.

Even though the method is focused on OAR DVH prediction, it could just as well be used for target DVH prediction (the signed distances from the target boundary are then negative).

\subsection{A Simple Example}

To further explain the algorithm, it will be demonstrated by a simple example with only a few data points. For clarity, the probability densities are estimated discretely instead of using a continuous density estimation method.

Figure 1a shows a stylized brain in 2D with a delineated target (red) and OAR (blue). From Figure 1a we can estimate the joint probability $p_{D, X}(d, x)$ of the dose $d$ and the signed distance $x$ from the target boundary to a certain OAR voxel (corresponding to step 1 in Algorithm 1)

$$
\begin{array}{ll}
p_{D, X}(13,2)=\frac{1}{3}, & p_{D, X}(13, \sqrt{5})=0 \\
p_{D, X}(12,2)=0, & p_{D, X}(12, \sqrt{5})=\frac{1}{3} \\
p_{D, X}(11,2)=0, & p_{D, X}(11, \sqrt{5})=\frac{1}{3} .
\end{array}
$$

Step 2 instructs us to also estimate the distance probability distribution $p_{X}(x)$

$$
p_{X}(2)=\frac{1}{3}, \quad p_{X}(\sqrt{5})=\frac{2}{3} .
$$

Finally, we calculate the conditional probability $p_{D \mid X}(d \mid x)=p_{D, X}(d, x) / p_{X}(x)$

$$
\begin{array}{llrl}
p_{D \mid X}(13 \mid 2) & =\frac{1 / 3}{1 / 3}=1, & & p_{D \mid X}(13 \mid \sqrt{5})=\frac{0}{2 / 3}=0 \\
p_{D \mid X}(12 \mid 2) & =0, & p_{D \mid X}(12 \mid \sqrt{5}) & =\frac{1 / 3}{2 / 3}=1 / 2 \\
p_{D \mid X}(11 \mid 2) & =0, & p_{D \mid X}(11 \mid \sqrt{5}) & =1 / 2 .
\end{array}
$$

We can now make predictions for a new case (indicated by *), for example the one seen in Figure 1b, where the target has an added pixel. We start by 


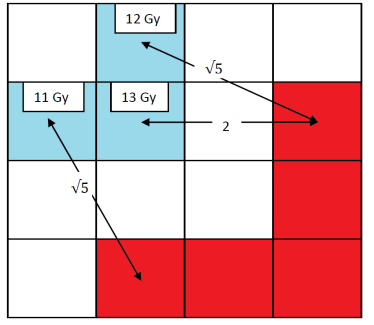

(a)

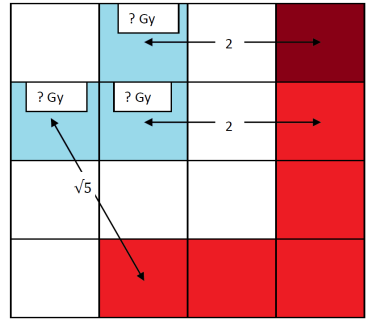

(b)

Figure 1: Stylized brain in 2D where blue pixels represent an OAR and red pixels a target. The arrows indicate the result of a distance transform between OAR pixels and target surface, meaning that the distance is measured between each OAR voxel and its closest target boundary voxel. The distances are indicated in the arrows. The case illustrated in Figure 1a is used for training and the dose, presented in the text box in each OAR pixel, is therefore known, whereas the case in Figure 1b is a new case for which the dose distribution is unknown. The dark red pixel indicates that the target of the new case has an added pixel.

estimating the distance distribution $p_{X}^{*}(x)$ for the new case (Algorithm 2, step 1)

$$
p_{X}^{*}(2)=\frac{2}{3}, \quad p_{X}^{*}(\sqrt{5})=\frac{1}{3} .
$$

The prediction of the dose distribution for the new patient can now be calculated by marginalization according to step 2 in Algorithm 2, written in discrete terms as $p_{D}^{*}(d)=\sum_{i} p_{D, X}\left(d \mid x_{i}\right) p_{X}^{*}\left(x_{i}\right)$.

$$
\begin{aligned}
& p_{D}^{*}(13)=p_{D \mid X}(13 \mid 2) p_{X}^{*}(2)+p_{D \mid X}(13 \mid \sqrt{5}) p_{X}^{*}(\sqrt{5})=0+1 \cdot \frac{2}{3}=\frac{2}{3} \\
& p_{D}^{*}(12)=p_{D \mid X}(12 \mid 2) p^{*}(2)+p_{D \mid X}(12 \mid \sqrt{5}) p_{X}^{*}(\sqrt{5})=\frac{1}{2} \cdot \frac{1}{3}+0=\frac{1}{6} \\
& p_{D}^{*}(11)=p_{D \mid X}(11 \mid 2) p_{X}^{*}(2)+p_{D \mid X}(11 \mid \sqrt{5}) p_{X}^{*}(\sqrt{5})=\frac{1}{2} \cdot \frac{1}{3}+0=\frac{1}{6} .
\end{aligned}
$$

Compare the above result to the dose distribution $p(d)$ that can be estimated for the training patient directly from Figure 1a

$$
p_{D}(13)=\frac{1}{3}, \quad p_{D}(12)=\frac{1}{3}, \quad p_{D}(11)=\frac{1}{3} .
$$

Since more of the OAR in the new case is closer to the target than in the training case, a larger part of the OAR is expected to receive a higher dose. The final predicted DVH for the new patient is found according to Equation (1). The predicted DVH can be observed in Figure 2.

In the example, only one training patient was used. In general you would want to use as many relevant patients as possible in the training set to bring in 


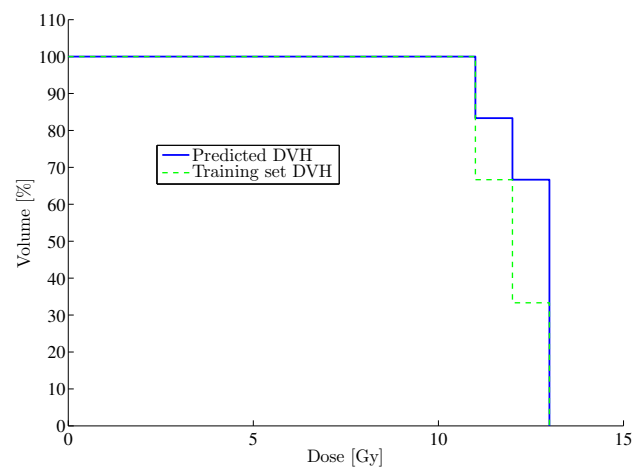

Figure 2: The predicted DVH for the new patient compared to the DVH for the training example. The predicted DVH reflects that as a larger part of the organ at risk is close to the tumor a larger part is also expected to receive a higher dose.

Table 1: Criteria used in the evaluation.

\begin{tabular}{lcc} 
Considered OAR & Volume $\left[\mathrm{cm}^{3}\right]$ & Threshold dose $[\mathrm{Gy}]$ \\
\hline Lung (left \& right) & $>1500$ & $<11.6$ \\
Lung (left \& right) & $>1000$ & $<12.4$ \\
Brain stem & $<0.5$ & $>10$
\end{tabular}

as much prior knowledge as possible, but the algorithm as such can in practice be used with a limited amount of data.

\subsection{Model Validation}

The method has been tested on 22 acoustic schwannoma patients treated with Gamma knife radiosurgery and 9 non-small cell lung cancer patients treated with stereotactic body radiation therapy (SBRT). The treatment plans were selected retrospectively and the clinically most relevant validation-replanning of plans flagged as inferior - was therefore not possible. Other researchers [1, 18, 25] have however shown the clinical significance of their algorithm in this way. As a proof of concept, we will in this study show that our method achieves a similar performance as those, clinically validated, methods.

We used Leave-One-Out Cross-Validation [15] (LOOCV) to test the performance of the algorithm, since this is an effective way of validating a model when the amount of data is limited [6]. We predicted DVHs for the brain stem of the acoustic schwannoma patients and for the lungs of the lung cancer patients. The predicted- and real DVHs were compared with respect to the evaluation criteria suggested in the AAPM report on stereotactic body radiation therapy [2]. The relevant criteria are summarized in Table 1. 


\subsubsection{Comparison with Previous Work}

To further validate the model, we have implemented the method proposed by Zhu et al. [28]. That is, a Support Vector Regression algorithm with a Radial Basis Function kernel, in our case implemented using LIBSVM [7]. The input consists of the OAR size, target size and 50 points on the $\mathrm{OVH}^{1}$. The output features are 50 points on the DVH. Both the 50 dimensional feature spaces of $\mathrm{OVH}$ points and DVH points are reduced to three feature dimensions each, using Principal Component Analysis. We have also standardized all the dimensions, by subtracting the mean and dividing by the standard deviation, generating zero mean and unit variance. For the data where the dimensionalities were reduced, we standardized the dimensions - as is customary - before performing the Principal Component Analysis. Just like Zhu et al. we set the tolerance parameter $\epsilon$ to 0.2. The $\gamma$-parameter of the Radial Basis Function and the error penalty parameter were determined by cross validation.

We have also implemented the method proposed by Appenzoller et al. [1]. First, a skew-normal probability distribution is fitted to the distribution of doses in voxels that are partitioned in $3 \mathrm{~mm}$ thick shells at particular distances from the target boundary. The dose distributions were estimated using standard histogram estimation with 100 bins. However, in order to fit the skew normal distribution to the dose distributions, we sub-sampled the histograms. The best sampling rate was determined for each sub-dose distribution by cross validation. Second, the means of the skew-normal parameters as functions of distance are fitted with polynomials. As in the work of Appenzoller et al., we used nonlinear least-squares to estimate the parameters of the skew-normal distribution. However, this method does not guarantee that the global optimum is found and in our implementation, outliers were manually removed before taking the mean of each fitting parameter. The degree of the polynomials was determined by cross validation. To perform the prediction, the probability distribution at each distance for the new patient's OAR is calculated by retrieving the fitting parameters - corresponding to each distance - from the polynomials. The dose distribution for the entire volume is found by multiplying each sub-dose distribution with the volume of the voxels at a certain distance and then summing the product. Since a skew-normal distribution is not able to describe multi-modal distributions, the distribution is estimated separately for in-field and out-offield voxels, before combining them. The in-field part of an OAR is defined as being between two boundary planes located 6 millimetres above and below the target, in accordance with the work of Appenzoller et al. Converserly, out-offield voxels are the OAR voxels outside these boundary planes. Note that this distinction is irrelevant for the Gamma knife patients because of the machine's design.

\footnotetext{
${ }^{1}$ In the referenced article [28] the term distance-to-target histogram (DTH) was used instead of OVH.
} 
Table 2: Average of point-wise differences between predicted DVHs and the real treatment DVHs. The numbers refer to the mean absolute differences in percent of the OAR volume at the threshold doses given in Table 1. The standard deviation from each mean is indicated after the \pm sign.

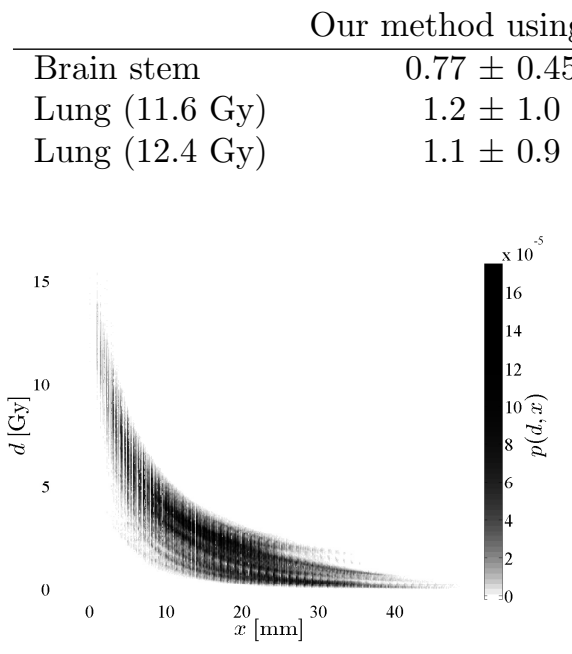

(a)

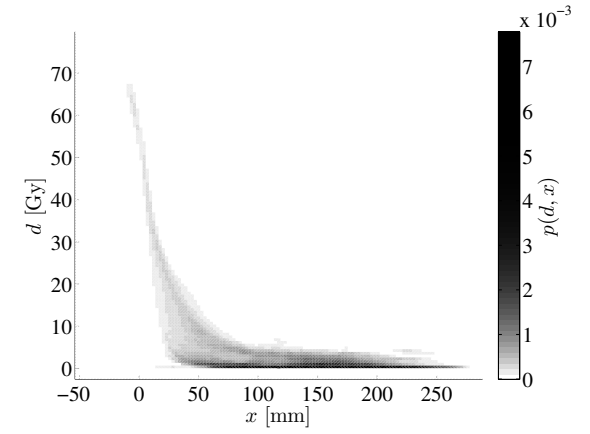

(b)

Figure 3: The joint probability density $p_{D, X}(d, x)$ for the dose $d$ and the signed distance $x$ between OAR voxels and target boundary. Figure 3a was estimated for the brain stem of 21 acoustic schwannoma patients treated with stereotactic radiosurgery and Figure $3 \mathrm{~b}$ was estimated for the lungs of 8 lung cancer patients treated with SBRT. Evidently, there is a strong correlation between the two variables.

\section{Results}

We have evaluated the proposed method for two different treatments (described in the subsections below). Kernel density estimation (KDE) was used to estimate probability densities with the signed distance to the target boundary as the predictive variable. Figure 3 shows the joint probability distribution estimated using KDE in two cases.

The predicted DVHs were compared with the DVHs predicted according to Appenzoller et al. [1] and Zhu et al. [28]. The DVHs from the real treatment were used as reference. A summary of point-wise differences between the predicted DVHs and the real treatment DVHs is given in Table 2.

\subsection{Stereotactic Radiosurgery Patients}

The method was tested on 22 acoustic schwannoma patients treated with stereotactic radiosurgery performed by the Gamma knife at a prescription dose of 13 


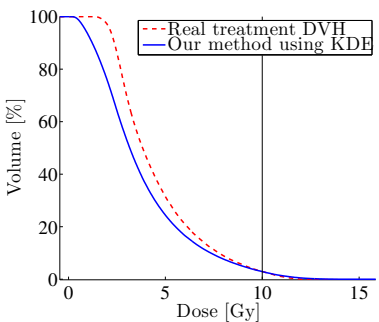

(a)

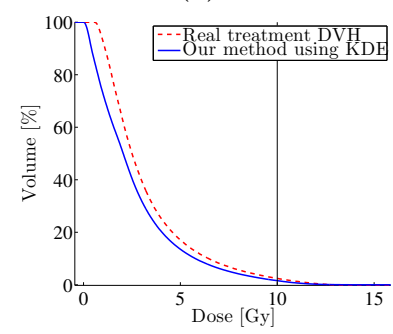

(d)

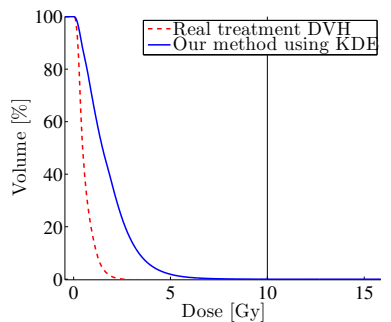

(b)

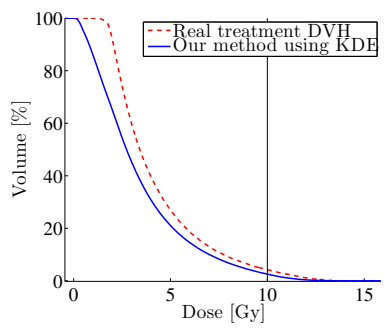

(e)

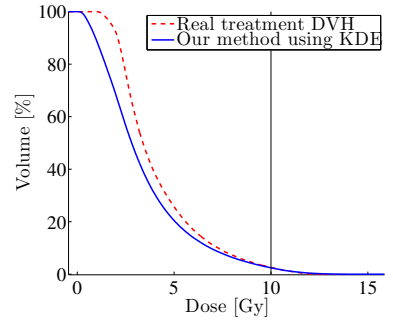

(c)

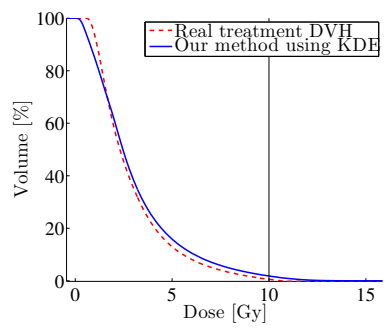

(f)

Figure 4: Six examples from the set of 22 acoustic schwannoma patients where the brain stem DVHs predicted using kernel density estimation (KDE) is compared to the real treatment DVH. These examples were selected based on how well the volumes predicted to receive at least 10 Gy matched the real treatment plan; the three closest matches at the top row and the three worst matches at the bottom row. The vertical line indicates the dose criteria 10 Gy at which the matching was made.

Gy at the $50 \%$ isodose level. The brain stem was the only OAR explicitly taken into account. The voxel size used for this set of patients was $1 \times 1 \times 1 \mathrm{~mm}^{3}$ and the signed distance was computed using the MATLAB function bwdist [16] with a Euclidean distance measure.

The brain stem DVH was predicted using the proposed method. Figure 4 shows the DVHs where the volumes predicted to receive at least 10 Gy matched the real treatment plan best and worst. Note that this matching criteria does not consider whether the entire predicted DVH is similar to the real one, instead it emphasizes a clinically relevant part of the DVH. This explains why the predicted DVH in Figure 4b is considered a good match: in this case the brain stem is located relatively far from the tumor making both DVHs effectively zero at the dose criteria.

Figure 5 compares the point-wise predictions for all the patients for all the methods discussed. Many of the values, both predicted and real, in this diagram are located close to zero. This is most likely caused by the fact that the dose criteria in many cases actually lies in the tail of the DVH. 


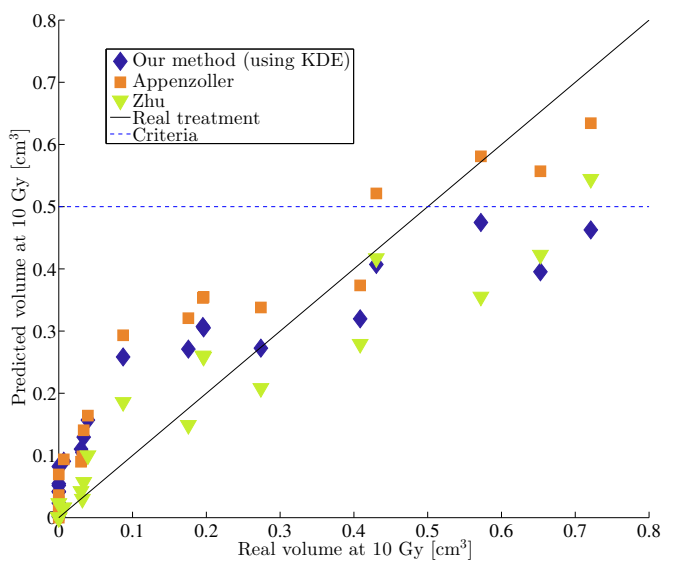

Figure 5: Predicted volume of the brain stem receiving at least 10 Gy for the stereotactic radiosurgery patients plotted against the volume receiving at least the same dose in the corresponding real treatment plans.

\section{$3.2 \quad$ SBRT Patients}

The method was also tested on 9 non-small cell lung cancer patients treated with a three-fraction course of stereotactic body radiation therapy (SBRT) at a prescription dose of 54 Gy at the $80 \%$ isodose level. The voxel size in this set of patients was $0.95 \times 0.95 \times 3 \mathrm{~mm}^{3}$. Since the voxels are anisotropic, the function bwdistsc [17] was used to compute the signed distances.

DVHs for the lungs predicted using KDE are shown in Figure 6. Figure 7 shows, for all patients and using all the methods discussed, the point-wise predictions of the lung volume receiving less than 11.6 Gy. From both figures, we can see that the predicted DVHs match well with the real DVHs and that the predictions of the different methods are similar.

\section{Discussion}

From Table 2 and figures 5 and 7 it is clear that -at clinically relevant points on the OAR DVHs - the various methods produce similar predictions. Since we cannot know if the treatment DVH is actually the best achievable DVH it is precarious to compare with it. On the other hand, it is reassuring that the proposed method behaves similarly to other, extensively validated, methods. This is perhaps expected considering that we have used the same predictive variable, namely the signed distance to the boundary of regions of interest, for all methods. As evident from Figure 3, this is not a bad choice: the correlation with the dose is striking. Nevertheless, a natural extension would be to include other variables - any variable affecting the clinical decision making could potentially 


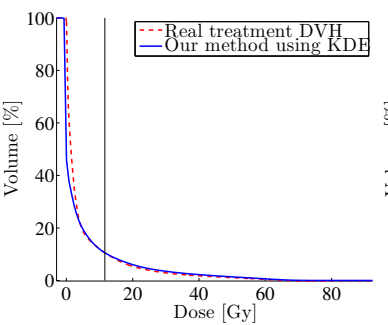

(a)

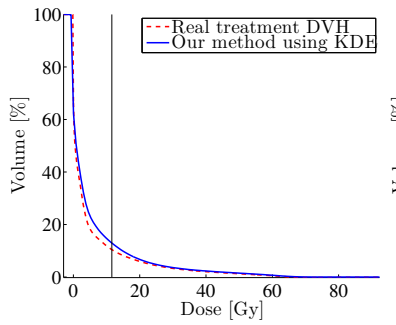

(d)

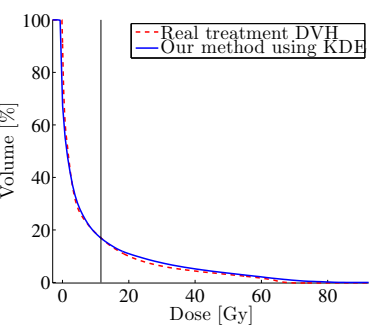

(b)

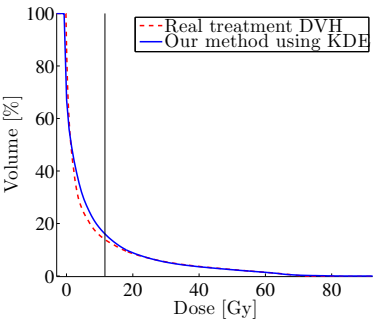

(e)

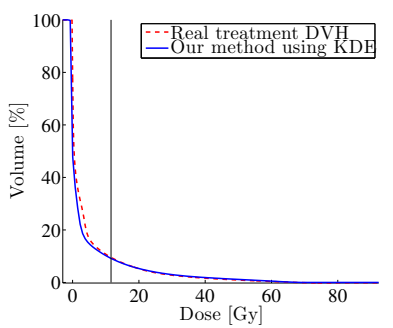

(c)

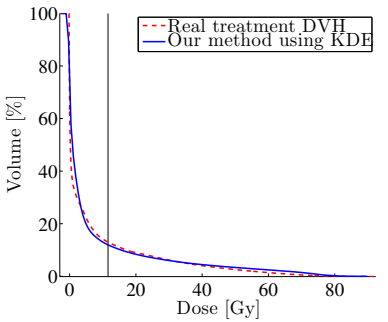

(f)

Figure 6: The above figures show six examples of lung DVHs from the 9 lung cancer patients where the DVHs predicted using kernel density estimation (KDE) are compared with the real treatment DVH. The selection of the DVHs was based on how well the volumes predicted to receive at most 11.6 Gy matched the real treatment plan; the three closest matches at the top row and the three worst matches at the bottom row. The dose criteria $11.6 \mathrm{~Gy}$, at which the matching was made, is indicated by the vertical line.

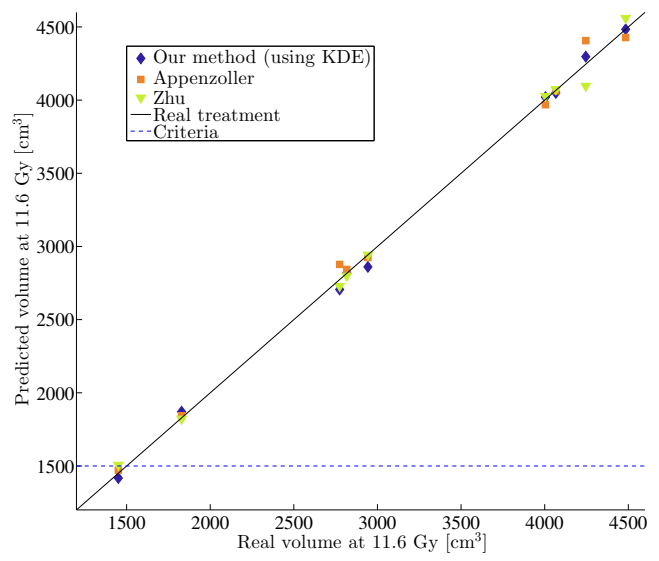

Figure 7: Predicted volume of the lungs receiving less than 11.6 Gy for the lung cancer patients plotted against the volume receiving less than that dose in the corresponding real treatment plans. 
be useful - but we defer such investigations to future work.

Table 2 also indicate that the mean absolute difference in volume at the specified dose criteria is less in the predicted brain stem DVHs, than in the predicted lung DVHs. One reason for this is that the brain stem, in contrast to the lungs, is a serial organ. The volumes of any hot spots are therefore by design small.

Non-parametric density estimation methods can have a high variance outside the domain of observed data. In cases where extrapolation might be necessary, a strong prior in the form of a particular parameterization, as in the method of Appenzoller et al., can be useful. On the other hand, specifying an appropriate parameterization can be difficult - if at all possible - when considering multiple variables or new types of variables. For instance, the assumption of an underlying skew-normal distribution makes the method unable to model multimodal distributions. This is why Appenzoller et al., in contrast to the KDE approach we have demonstrated, have to make the distinction between in-field and out-of-field voxels.

Without further restrictions on the regressed DVH, the method of Zhu et al. [28] may predict "DVHs" that violate two intrinsic properties of a DVH: that it must be non-increasing and take values only in the range $[0,1]$. However, since this appears to occur in the low-dose regions it may, depending on the application, be insignificant. On the other hand, the method is flexible in the sense that it is able to incorporate multiple input variables in a straightforward manner.

The proposed method in itself does not place any restrictions on the resolution of the dose grid, however, it may affect the accuracy of the density estimation method (cf. the choice of bin width in a histogram). We consistenly used the voxel sizes present in the treatment plans.

We envision that, depending on the training data provided, the method could be of use in at least two different cases. Having a training set consisting of exceptionally high quality plans (a "gold standard") the resulting predictions would be of the best possible DVHs that could be achieved. On the other hand, if the training set consists of typical plans created at a clinic, the predictions would reflect the average case DVHs that could be expected at the clinic. The first case would be of interest when planning new treatments whereas the second would be useful in retrospective evaluations.

\section{Conclusion}

We propose a method for DVH prediction that respects the intrinsic properties of DVHs while making minimal assumptions. At the same time, it is more general than previously proposed methods in the sense that it can be used with any number of input variables and any density estimation method. We demonstrated, on two different sets of patients, that when trained on the same input data as two previously proposed methods the predictions are similar.

To conclude, the method could be used for quality assurance and possibly 
also to guide DVH-based treatment plan optimization. It is also conceivable to use it for large scale comparisons of systematic differences between treatment centers or planners.

\section{Acknowledgements}

The authors would like to thank Dr Xiao Han for valuable discussions. The research was supported by the Swedish Research Council (grant 2012-4281) and the Linneaus center CADICS. The work is part of the Swedish testbed for innovative radiotherapy.

\section{References}

[1] L. M. Appenzoller, J. M. Michalski, W. L. Thorstad, S. Mutic, and K. L. Moore. Predicting dose-volume histograms for organs-at-risk in IMRT planning. Medical Physics, 39:7446-7461, 2012.

[2] S. H. Benedict, K. M. Yenice, D. Followill, J. M. Galvin, W. Hinson, B. Kavanagh, P. Keall, M. Lovelock, S. Meeks, L. Papiez, T. Purdie, R. Sadagopan, M. C. Schell, B. Salter, D. J. Schlesinger, A. S. Shiu, T. Solberg, D. Y. Song, V. Stieber, R. Timmerman, W. A. Tomé, D. Verellen, L. Wang, and F.-F. Yin. Stereotactic body radiation therapy: The report of AAPM Task Group 101. Medical Physics, 2010.

[3] C. M. Bishop. Pattern Recognition and Machine Learning. Springer, 2006.

[4] J. Bohsung, S. Gillis, R. Arrans, A. Bakai, C. De Wagter, T. Knöös, B. J. Mijnheer, M. Paiusco, B. A. Perrin, H. Welleweerd, and P. Williams. IMRT treatment planning - A comparative inter-system and inter-centre planning exercise of the QUASIMODO group. Radiotherapy and Oncology, 76(3):354-361, 2005.

[5] Z. I. Botev, J. F. Grotowski, and D. P. Kroese. Kernel density estimation via diffusion. The Annals of Statistics, 38(5):2916-2957, 2010.

[6] G. Cawley and N. Talbot. On over-fitting in model selection and subsequent selection bias in performance evaluation. Journal of Machine Learning Research, 11:2079-2107, 2010.

[7] C.-C. Chang and C.-J. Lin. LIBSVM: A library for support vector machines. ACM Transactions on Intelligent Systems on Technology, 2:27:127:27, 2011. Software available at http://www.csie.ntu.edu.tw/ cjlin/ libsvm.

[8] V. Chanyavanich, S. K. Das, W. R. Lee, and J. Y. Lo. Knowledge-based IMRT treatment planning for prostate cancer. Medical physics, 38(5):2515$2522,2011$. 
[9] K. C. Ciesielski, J. K. Udupa, X. Chen, and G. J. Grevera. Linear time algorithms for exact distance transform elaboration on Maurer et al. algorithm. Journal of Mathematical Imaging and Vision, 39(3):193-209, 2011.

[10] I. Das, C.-W. Cheng, K. L. Chopra, R. K. Mitra, S. P. Srivastava, and E. Glatstein. Intensity-modulated radiation therapy dose prescription, recording, and delivery: patterns of variability among institutions and treatment planning systems. Journal of the National Cancer Institute, 100(5):300-307, 2008.

[11] J. Deasy. Multiple local minima in radiotherapy optimization problems with dose-volume constraints. Medical physics, 24(7):1157-1161, 1997.

[12] S. Gillis, C. De Wagter, J. Bohsung, B. A. Perrin, P. Williams, and B. J. Mijnheer. An inter-centre quality assurance network for IMRT verification: Results of the ESTRO QUASIMODO project. Radiotherapy and Oncology, $76: 340-353,2005$.

[13] D. Good, J. Lo, W. R. Lee, Q. J. Wu, F.-F. Yin, and S. K. Das. A knowledge-based approach to improving and homogenizing intensity modulated radiation therapy planning quality among treatment centers: An example application to prostate cancer planning. International Journal of Radiation Oncology* Biology* Physics, 87(1):176-181, 2013.

[14] M. Kazhdan, P. Simari, T. McNutt, B. Wu, R. Jacques, M. Chuang, and R. Taylor. A shape relationship descriptor for radiation therapy planning. In Medical Image Computing and Computer-Assisted Intervention-MICCAI 2009, volume 5762 of Lecture Notes in Computer Science, pages 100108. Springer Berlin Heidelberg, 2009.

[15] P. A. Lachenbruch and M. R. Mickey. Estimation of error rates in discriminant analysis. Technometrics, 10:1-11, 1968.

[16] C. Maurer, Q. Rensheng, and V. Raghavan. A linear time algorithm for computing exact Euclidean distance transforms of binary images in arbitrary dimensions. IEEE Transactions on Pattern, 25(2):265-270, 2003.

[17] Y. Mishchenko. A function for fast computation of large discrete euclidean distance transforms in three or more dimensions in Matlab. Signal, Image and Video Processing, 2013.

[18] K. Moore, L. Appenzoller, J. Tan, J. Michalski, W. Thorstad, and S. Mutic. Clinical implementation of dose-volume histogram predictions for organsat-risk in IMRT planning. In Journal of Physics: Conference Series, volume 489, page 012055. IOP Publishing, 2014.

[19] K. L. Moore, R. S. Brame, D. A. Low, and S. Mutic. Experience-based quality control of clinical intensity-modulated radiotherapy planning. International Journal of Radiation Oncology* Biology* Physics, 81(2):545-551, 2011. 
[20] K. P. Murphy. Machine Learning: A Probabilistic Perspective. The MIT press, 2012.

[21] P. Ram and A. G. Gray. Density estimation trees. In Proceedings of the 17th ACM SIGKDD international conference on Knowledge discovery and data mining, pages 627-635. ACM, 2011.

[22] R. Siegel et al. Cancer treatment and survivorship statistics, 2012. CA: a cancer journal for clinicians, 62(4):220-241, 2012.

[23] S. Thrun, W. Burgard, and D. Fox. Probabilistic Robotics. The MIT press, 2005.

[24] J. Toriwaki and H. Yoshida. Fundamentals of Three-dimensional Digital Image Processing. Springer, 2009.

[25] B. Wu, F. Ricchetti, G. Sanguineti, M. Kazhdan, P. Simari, M. Chuang, R. Taylor, R. Jacques, and T. McNutt. Patient geometry-driven information retrieval for IMRT treatment plan quality control. Medical Physics, 36(12):5497-5505, 2009.

[26] B. Wu, F. Ricchetti, G. Sanguineti, M. Kazhdan, P. Simari, R. Jacques, R. Taylor, and T. McNutt. Data-driven approach to generating achievable dose-volume histogram objectives in intensity-modulated radiotherapy planning. International Journal of Radiation Oncology* Biology* Physics, 79(4):1241-1247, 2011.

[27] M. Zarepisheh, M. Shakourifar, G. Trigila, P. S. Ghomi, S. Couzens, A. Abebe, L. Noreña, W. Shang, S. Jiang, and Y. Zinchenko. A momentbased approach for DVH-guided radiotherapy treatment plan optimization. Physics in Medicine and Biology, 58(6):1869, 2013.

[28] X. Zhu, Y. Ge, T. Li, D. Thongphiew, F. Yin, and Q. Jackie Wu. A planning quality evaluation tool for prostate adaptive IMRT based on machine learning. Medical Physics, 38:719-26, 2011.

[29] Y. Zinchenko, T. Craig, H. Keller, T. Terlaky, and M. Sharpe. Controlling the dose distribution with gEUD-type constraints within the convex radiotherapy optimization framework. Physics in Medicine and Biology, 53(12):3231-3250, 2008. 